Classic signs revisited

\title{
The Babinski reflex
}

\author{
J van Gijn
}

\section{Summary}

The plantar response is a reflex that involves not only the toes, but all muscles that shorten the leg. In the newborn the synergy is brisk, involving all flexor muscles of the leg; these include the toe 'extensors', which also shorten the leg on contraction and therefore are flexors in a physiological sense. As the nervous system matures and the pyramidal tract gains more control over spinal motoneurones the flexion synergy becomes less brisk, and the toe 'extensors' are no longer part of it. The toes then often go down instead of up, as a result of a segmental reflex involving the small foot muscles and the overlying skin, comparable to the abdominal reflexes. With lesions of the pyramidal system, structural or functional, this segmental, downward response of the toes disappears, the flexion synergy may become disinhibited and the extensor hallucis longus muscle is again recruited into the flexion reflex of the leg: the sign of Babinski.

A true Babinski sign denotes dysfunction of the pyramidal tract, and should be clearly distinguished from upgoing toes that do not belong to the flexion synergy of the leg. Correct interpretation of the plantar response depends only to a minor degree on the method or site of stimulation of the foot. It is therefore most important to assess the response in the entire leg.

Keywords: Babinski reflex

University Department of Neurology, Academisch Ziekenhuis, PO Box 85500 , 3508 GA Utrecht, The Netherlands $\mathrm{J}$ van Gijn

Accepted 28 June 1995
The information that may be deduced from scratching a patient's sole, is as important as a diagnostic sign as it is simple to elicit. When the great toe moves upward (sign of Babinski), this signifies, as everybody knows, a disturbance of the pyramidal tract. This explains why few patients with neurological symptoms can avoid having their plantar reflexes examined - often to their great surprise, or even alarm if the trouble is in the head. Unfortunately the reality is less simple than the theory. Often it is difficult to decide whether the great toe actually does go up: the toe movements may be slight, vacillate between up and down, seem down one day but up the next, or be interrupted by voluntary withdrawal movements. It is therefore no great surprise that such difficult plantar responses give rise to wide variations between doctors, or even between different occasions with the same observer. Under these circumstances the interpretation may be influenced by the physician's previous expectations. ${ }^{1}$ Since it is obviously important for the diagnosis in individual patients whether or not a lesion of the pyramidal system exists, there is a need for criteria that are both reliable and simple. The guidelines that follow have not only been validated in practice, ${ }^{2,3}$ but are also founded on physiological principles.

\section{Physiology}

The quintessence is that the plantar reflex entails more movements than just those of the toes. In most mammals the limbs are automatically retracted on painful stimulation, a defensive reflex subserved by polysynaptic pathways in the spinal cord. The reaction is more pronounced in hind limbs, because the forelimbs are under more direct control of the brain. The reflex is stronger as the stimulus is more distal. Not only the skin but also deeper structures contain receptors that may release this movement. Around the turn of the century Sherrington extensively analyzed this phenomenon in animals; he called it the flexion reflex, because the effect consisted of the activation of all muscles that shortened the limb (physiological flexors). Somewhat surprisingly, the toe extensors form part of this shortening synergy: the (anatomical) toe extensors are flexor muscles in a physiological sense, and vice versa.

The reflex effects in the human leg on stimulation of the sole are completely analogous to those in animals. This is especially clear in neonates, provided the plantar surgace is not stimulated too gently, in which case a grasp reflex occurs. ${ }^{4}$ In the neonate there are two differences with respect to the normal adult: the entire flexion synergy is much more brisk and, more important, the toes are part of the synergy: they go up at the same time that flexion occurs in the ankle, knee and hip. Again, for the anatomist these are extensory movements of the toes, for the physiologist, flexor movements. That the movement is greatest in the great toe results from the structure of the metacarpo-phalangeal joints; the little toes simply cannot move up that far.

As the pyramidal system matures and gains more control over spinal motoneurones the flexion reflex changes in two respects. The first and more important change is that after one or two years the toes are no longer part of the flexion synergy. The second is that the entire flexion reflex becomes less brisk; in most adults all that is left of the composite movements is a subtle contraction of the proximal muscles, particularly of the musculus tensor fasciae latae, under the skin of the later part of the thigh. In some adults the remaining part of the flexion reflex stays brisk or, conversely, disappears; only asymmetry is truly pathological, as is the case with muscle stretch reflexes. Not only do the toes of older children and adults no longer go up on stimulation of the sole, in most cases the movement is replaced by an opposite, downward displacement. This reflex is mediated by the short toe flexors (anatomical term) of the sole, and has nothing to do with flexion synergy; it is a purely cutaneous reflex, related to a single segment of the spinal cord. The downward movement may predominate in the great toe or the smaller toes, depending on whether the medial or the lateral part of the sole is stimulated. Absence of the downward toe response is not pathological unless there is a marked difference between the two sides. 


\section{The flexion reflex}

- on stimulation of distal skin, or deeper structures

- shortening of lower limb in hip, knee, ankle and toes

- toe 'extensors' are flexors in a physiological sense

\section{Role of the pyramidal tract}

- decreases entire flexion synergy

- inhibits participation of toe 'extensors'

- dysfunction of pyramidal tract restores 'neonatal' situation

\section{Lesions of the pyramidal system}

The function of the pyramidal tract may not only be distrubed by structural lesions - of myeline sheaths, axons, or both - but also by metabolic factors, for example, hypoglycaemia or an epileptic seizure. In these cases the flexion reflex may become brisker, by disinhibition. The sign of Babinski emerges when the dysfunction involves those pyramidal fibres that project upon motoneurones of foot muscles; the great toe may again be recruited into the flexion synergy in the leg. In these cases there is almost always some degree of motor deficit in the footoften in the form of weakness, but sometimes evident only from a decreased ability to perform rapid foot movements (eg, when the patient is asked to tap rapidly against the bottom of the bed or the examiner's hand). ${ }^{5}$

Not all disturbances of the pyramidal system are associated with a Babinski sign, even in cases where the descending fibres to the foot muscles are clearly involved. The explanation for the lack of a Babinski reflex should be sought in a dysfunction of the segmental reflex pathways, the simplest being a pressure palsy of the peroneal nerve. In other cases there is temporary inexcitability of spinal motoneurones, most often by a transverse lesion of the cord ('spinal shock'), but sometimes after acute brain lesions. ${ }^{5}$ Then it is not only the up-going toe sign that is lacking, but the entire flexion synergy of the leg.

The muscle responsible for the up-going toe sign is located in the leg: the musculus extensor hallucis longus. ${ }^{6}$ This has practical implications. It is also of great practical importance that an up-going toe sign rarely occurs without accompanying reflex activity in more proximal flexor muscles of the leg. Babinski himself emphasized from the outset the intimate relationship between his sign and the shortening movements in other leg muscles. Yet subsequently, many had eyes for the toes alone, and so got into trouble.

\section{Positioning and stimulation}

To start with, all leg muscles should be visible and in a relaxed state. This implies that the entire leg should be uncovered (removing socks is not enough), and that the patient should be supine, on a couch or in a bed (hoisting up the leg of a seated patient is asking for trouble). The patient should be warned that the sole is going to be scratched, and that it is important that the limbs remain as floppy as possible (use of the term 'relax' is almost invariably followed by the very muscle contractions one wishes to avoid).

It is the site of stimulation, the intensity of stimulation, and even the object used for stimulation (from inverted patella hammer to shark's tooth or Bentley key) that has received by far the most attention in relation to difficulties in interpreting the plantar response. Any instrument will do provided it is not too sharp and has not been used on other patients. My own favourite instrument is the end of a thin wooden stick, of the type that may have a piece of cotton wool at the other end. Any part of the leg can be stimulated, but the chance of producing an up-going toe response in the presence of pyramidal tract dysfunction is greatest if the lateral plantar border is chosen, rather than the medial side, which overlies the flexor hallucis brevis muscle. Stimulation at that site is therefore more likely to make the toe go down. Ideally the stimulus should continue along the lateral border of the sole, and should be rather slow. ${ }^{7}$ On the other hand, repeated interference by voluntary movements the patient is unable to suppress may make it desirable to use a shorter stimulus, or a stimulus at a different site. Stroking the lateral dorsum of the foot (figure 1) is often helpful in such cases, and may even be more effective in producing a Babinski sign. This is also because irritation of the sole can give rise to voluntary clawing of the toes.

\section{Interpretation of the plantar response}

The following three rules governing the relationship between the Babinski sign and the flexion synergy were determined in a study of patients with equivocal plantar respones in whom clinical and electromyographic features were compared. ${ }^{2}$

Upward movement of the great toe is pathological only if caused by contraction of the extensor hallucis longus muscle

In most patients tightening of the tendon can be seen or at least felt, on the dorsum of the great toe and the foot (figure 1). The hallux may move upwards without contraction of the extensor hallucis longus muscle on dorsiflexion of the foot as a whole, a movement that often occurs as part of the flexion reflex and which is not pathological in itself. ${ }^{6}$ In addition, the great toe may go up merely after having flexed, and a brisk flexion synergy of the leg may conceal this initial 
Figure 1 Babinski sign (in this case evoked by stroking the lateral part of the dorsum rather than of the sole, in order to avoid voluntary withdrawal by ticklishness). Note the protrusion of the tendon of the extensor longus muscle on the dorsum of the great toe and the foot
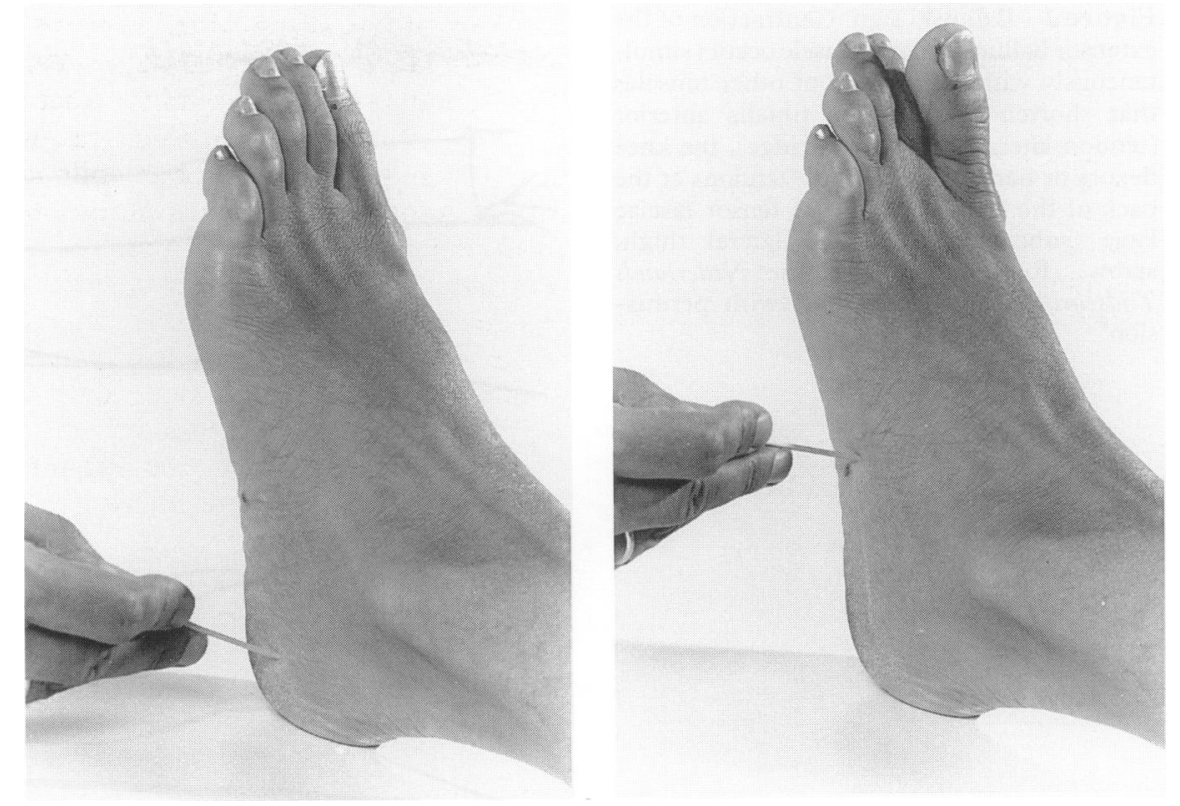

movement. Finally, an illusion of the big toe going up may be created if the other toes go gently down, the big toe being actually immobile.

Contraction of the extensor hallucis longus muscle is pathological only if it occurs synchronously with reflex activity in other flexor muscles

The flexion synergy may be weak in some subjects, but as contraction of the tensor fasciae and hamstring muscles constitutes the minimal response, ${ }^{8}$ tightening of these muscles occurs at the same time as a true Babinski response. Whenever one examines the plantar response it is extremely important to look not only at the toe itself but also at the outer thigh and at the tendons of the hamstring muscles, as a reference for what is part of the flexion synergy and what is not (figure 2). Sometimes it may seem that the up-going toe is the minimal response of the flexion reflex, but one should keep in mind that the big toe is lighter than the foot, leg or thigh, and palpation of the hamstring tendons may uncover small degrees of contraction that were not readily visible. ${ }^{8}$ In most cases, however, the flexion synergy will lead to some displacement of the leg, and the examiner can not only see but also feel this with the hand that supports the foot while the other hand stimulates it.

A true up-going toe sign is reproducible, unlike voluntary withdrawal of the toes To avoid (more or less) voluntary reactions in patients with ticklish feet, the first useful measure is to tell the patient what is going to happen. Furthermore, one should adapt the stimulus as much as possible to what the patient can tolerate. Little is gained by faithfully completing a textbook manoeuvre while the patient is writhing on the couch. In such cases it often helps to stroke the lateral dorsum of the foot rather than the lateral sole (figure 1). One cannot see the normal downard response of the big toe, but the main purpose of the test is to see whether or not there is a Babinski sign. If stroking the full length of the dorsum is still too much for the patient, repeating stroking of only a few centimetres of skin will, in the case of flexion reflex, produce an approximately similar fraction of the synergy each time, whereas volumtary retraction will be abolished, or inconstant. Fatigue of the Babinski sign is extremely uncommon. Finally, voluntary withdrawal does not involve the tensor fasciae latae, and may preceed or outlast the stimulus.

\section{Myths dispelled}

The Babinski sign is so venerable that it has become the subject of $i$ dées recues that are, on closer scrutiny unfounded.

'The stimulus should be painful.' This is incorrect, as the necessary intensity depends on the degree of disinhibition of the flexion reflex. In some patients a mere touch anywhere on the leg is sufficient for releasing a brisk withdrawal of the entire leg, including the up-going toe sign. 
Figure 2 Babinski sign. Contraction of the extensor hallucis longus muscle occurs simultaneously with contraction of other muscles that shorten the leg: the tibialis anterior (tendon lateral to the tibial ridge), the knee flexors or hamstring muscles (tendons at the back of the knee), and the $M$ tensor fasciae latae (subcutaneous in the lateral thigh; arrow). Reproduced from the Nederlands Tijdschrift voor Geneeskunde, with permission $^{9}$

\begin{tabular}{|l|}
\hline Dispelled myths \\
\hline - stimulates need not be painful \\
- different sites of stimulation: same \\
flexion reflex \\
- eponyms serve only to torture \\
students \\
- first toe movement may or may not \\
be the response that counts \\
- Babinski sign may be slow or fast \\
panning of the toes is not a \\
prequisite \\
\hline
\end{tabular}

1 Van Gijn J, Bonke B. Interpretation of plantar reflexes: biasing effects of other signs and symptoms. I Neurol Neurosurg Psychiatry 1977; 40: 787-9.

2 Van Gijn J. Equivocal plantar responses: a clinical and electromyographic study. $\mathcal{F}$ Neurol Neurosurg Psychiatry 1976; 39: 275-82.

3 Raijmakers PGHM, Castro Cabezas M, Smal JA, Van Gijn J. Teaching the plantar reflex. Clin Neurol Neurosurg 1991; 93: 201-4.



'In doubtful cases one should apply variants of the Babinski sign.' Indeed the up-going toe sign can be elicited from many different sites at the foot or even the leg, but the physician (or student!) should not be deterred by the variety of eponyms. Wherever one scratches or pinches, any ensuing response of the toes is part and parcel of the flexion synergy of the lower limb. Any stimulus is legitimate as long as it is not applied more distally than the ball of the foot, as this may produce false-positive results.

'The movement should occur at the metatarsophalangeal joint.' The rationale of this criterion is to exclude passive upward movements of the great toe, merely by dorsiflexion of the foot. But a more sensitive method than inspecting the joint is to concentrate on the tendon of the extensor hallucis longus muscle (figure 1), from which the movement originates. This tendon has its insertion at the terminal phalanx; it may tighten even without any visible displacement of the toe.

'The movement should be the first'. It is difficult to see why a patient cannot make a voluntary movement before the onset of reflex action. Sometimes a trace of a downward response precedes the recruitment of the great toe into the flexion reflex synergy.

'The movement should be slow'. This view is echoed by many textbooks. But what is the definition of slowness? Moreover, reports abound of brisk reflexes and Babinski signs.

'In doubtful cases the 'fan sign' is a useful measure'. Fanning of the toes is of historical interest only. Babinski described this sign a few years after his discovery of the up-going toe sign, but he added that it may also occur in normal subjects. It may cause errors not only of observation but also of interpretation if more attention is paid to this phenomenon that it deserves.

In conclusion, the real question in interpreting the plantar response is not whether the great toe goes up or not, but whether an up-going toe is a pathological response. For an appropriate answer the method of watching is much more important than the method of scratching.

4 Dietrich HF. A longitudinal study of the Babinski and plantar grasp reflexes in infancy. $A m$ Dis Child 1957; 94: 265-71.

5 Van Gijn J. The Babinski sign and the pyramidal syndrome. F Neurol Neurosurg Psychiatry 1978 41: $865-73$.

6 Van Gijn J. Babinski response: stimulus and effector. $\mathcal{F}$ Neurol Neurosurg Psychiatry 1975; 38: $180-6$.
7 Dohrmann GJ, Nowack WJ. The upgoing grea toe - optimal method of elicitation. Lancet 1973 i: $339-41$

8 Walshe FMR. The physiological significance of the reflex phenomena in spastic paralysis of the lower limbs. Brain 1914; 37: 269-336.

9 Van Gijn J, van Crevel H, De voetzoolreflex. Ned Tijdschr Geneeskd 1978; 122: 1299-302. 